\title{
Ultralow Surface Tension Solvents Enable Facile COF Activation with Reduced Pore Collapse
}

\author{
Dongyang Zhu, ${ }^{\dagger}$ and Rafael Verduzco ${ }^{*},+,+$ \\ $\uparrow$ Department of Chemical and Biomolecular Engineering, Rice University, 6100 Main Street, MS-362, \\ Houston, Texas 77005, United States \\ †Department of Materials Science and Nanoengineering, Rice University, 6100 Main Street, MS-325, \\ Houston, Texas 77005, United States
}

ABSTRACT. Covalent Organic Frameworks (COFs) are organic, crystalline, highly porous materials attractive for applications such as gas storage, gas separations, catalysis, contaminant adsorption and membrane filtration. Activation of COFs removes adsorbed solvents and impurities, but common methods for COF activation can result in collapse of porous structure and loss of accessible surface areas. Here, we present a study of the impact of solvent surface tension on the activation process and demonstrate that activation using the ultralow surface tension solvent perfluorohexane $(\mathrm{PFH})$ is simple and effective for a range of COF materials. We synthesized six different imine-based COFs through imine condensation reactions between tris(4-aminophenyl) benzene (TAPB) or 2,4,6-tris(4-aminophenyl)-1,3,5-triazine (TAPT) and multi-functional di- and tri-benzaldehydes with different aromatic substituents. For each COF, we performed a solvent wash followed by vacuum drying using six solvents varying in surface tension from $11.9-72.8 \mathrm{mN} \mathrm{m}^{-1}$. Through powder X-ray diffraction (PXRD) measurements combined with nitrogen adsorption and desorption analysis, we found that some COF chemistries readily lost their porosity during activation with higher surface tension solvents while others were more robust. However, all COFs could be effectively activated using PFH to produce materials with excellent crystallinity and 
high surface areas, comparable to those for samples activated using supercritical $\mathrm{CO}_{2}$. This work demonstrates that the solvent surface tension used during activation has a strong impact on potential pore collapse, and activation using PFH provides a simple and effective activation method to produce COFs with excellent crystallinities and pore structures.

KEYWORDS: COFs, activation, ultralow surface tension solvents, perfluorohexane, pore collapse

\section{INTRODUCTION}

Covalent organic frameworks (COFs) are organic, crystalline, and highly porous materials linked by dynamic covalent bonds ${ }^{1}$. They have very high accessible surface areas and regular pore channels ${ }^{2}$, similar to other framework materials such as metal organic frameworks (MOFs) ${ }^{3}$ and zeolites ${ }^{4}$. Due to their structural tunability, crystallinity, and tailorable pore chemistry, COFs are attractive for various applications including gas storage $e^{5,6}$, gas separations ${ }^{7,8}$, catalysis ${ }^{9-11}$, contaminant adsorption ${ }^{12,13}$ and membrane filtration $7,14,15$.

Among various possible COF chemistries, COFs with imine linkages are of particular interest because of their excellent physical and chemical stabilities ${ }^{16}$. Significant efforts have been devoted to synthesizing imine COFs, including solvothermal reactions catalyzed by acetic acid ${ }^{1,2,10,16}$, microwave ${ }^{17,18}$ or sonication $^{19}$ assisted synthesis, room temperature synthesis using metal triflate ${ }^{20}$, mechanical grind synthesis assisted by 4-methylbenzenesulfonic acid ${ }^{21}$, among others. All these methods promote reversible reactions and facilitate the error-checking process in order to obtain highly crystalline imine COFs.

To isolate crystalline, porous, and dry COFs with accessible pores, adsorbed solvents and impurities such as unreacted monomers or oligomers must be removed. The process of removing these impurities and yielding dry COFs is generally termed activation, ${ }^{22,23}$ and activation is arguably as important as synthesis $^{22,23}$ but has received far less attention. Proper activation can effectively remove unreacted small molecules or generated oligomers, adsorbed solvents, and other guest molecules while maintaining COF crystallinity and pore structure ${ }^{22-27}$. The simplest and most commonly used activation process for COFs 
is solvent washing followed by vacuum drying ${ }^{1,22,23}$. However, this often produces materials with poor crystallinity, low surface areas, and collapsed pores ${ }^{22,23,28}$. For example, a TPB-TP COF obtained after washing with THF and vacuum drying at $120^{\circ} \mathrm{C}$ had a surface area of just $148 \mathrm{~m}^{2} \mathrm{~g}^{-128}$, far lower than the theoretical surface area ${ }^{29}$.

Activation processes for MOFs have been more extensively studied, and a number of approaches to MOF activation have been developed that do not compromise the structural integrity and porosity ${ }^{24-27}$. Some MOFs such as MIL- $101^{24}$ and UIO- $66^{24}$ are thermally and chemically stable, and these retained surface areas of $4100 \mathrm{~m}^{2} \mathrm{~g}^{-1}$ and $1070 \mathrm{~m}^{2} \mathrm{~g}^{-1}$, respectively, after simple heat and vacuum treatment. However, other MOFs lose crystallinity and porosity under similar activation procedures because their coordination bond strength cannot offset the strong surface tension and capillary forces induced by adsorbed solvents undergoing liquid-to-gas phase transitions ${ }^{24,25}$. For these MOFs, gentler activation methods such as washing with supercritical $\mathrm{CO}_{2}\left(\mathrm{ScCO}_{2}\right)$ can be used to remove adsorbed organic solvents without degrading their structure. $\mathrm{ScCO}_{2}$ will transition to the gas phase upon decreasing the pressure with minimal capillary forces exerted on the $\mathrm{MOF}^{24,25}$. Other strategies to minimize capillary forces during activation have also been developed and applied to MOFs ${ }^{26,27}$. For example, using low boilingpoint solvents to wash followed by low temperature vacuum drying effectively activated MOF-5 and produced dry materials with a Langmuir surface area as high as $2900 \mathrm{~m}^{2} \mathrm{~g}^{-126}$. In another approach, two isoreticular $\mathrm{Cu}$ paddlewheel-based MOFs were effectively activated by benzene washing and freeze drying $^{27}$.

However, there has not been comparable investigation and development of proper activation procedures for COFs. Two recent studies ${ }^{22,23}$ demonstrated the importance of proper activation for COFs and reported innovative methods for COF activation. Sick et al. ${ }^{22}$ systematically studied the structural changes of COFs in response to different guest molecules and employed $\mathrm{ScCO}_{2}$ activation as an effective way to isolate imine-based COFs with long-range ordering and surface areas higher than $1000 \mathrm{~m}^{2} \mathrm{~g}^{-1}$. By comparison, the same COFs activated with dioxane only exhibited short-range ordering and limited surface area. In another recently reported approach ${ }^{23}$, imine-based COFs were activated under nitrogen flow after 
sequential washing using a series of organic solvents. This method minimized capillary forces during solvent removal.

The sensitivity of COFs to different activation processes also depends on COF chemistry ${ }^{22,26,28}$. Sick et $a l .{ }^{22}$ noted that some COF chemistries were 'fragile' and readily lost crystallinity during harsh activation processes, while other, more robust COFs maintained crystallinity under a variety of activation processes. The fragility was attributed to weak interlayer $\pi-\pi$ interactions ${ }^{22,23}$, resulting in pore collapse when polar solvents (e.g., dimethylformamide, dioxane) were evacuated from the pore cavities. Molecular engineering strategies have been employed to improve COF stability, such as the use of molecular docking sites $^{30}$, increased intralayer hydrogen bonding ${ }^{31}$, or using fluorine atoms to increase the strength of $\pi-\pi$ interactions ${ }^{32}$. However, there is no a priori method to identify fragile and robust COFs based on their structures, so conventional vacuum activation cannot be readily employed for effective activation of COFs with unknown fragility. Furthermore, while $\mathrm{ScCO}_{2}$ drying and nitrogen-flow drying are effective for producing crystalline COFs, these methods may not be readily accessible or scalable to larger quantities of COFs. During the preparation and analysis of new COF samples, a simpler approach to activation would be desirable. Therefore, we were motivated to develop an effective and simple activation method that involved only solvent washing and vacuum drying. We hypothesized that this could be achieved by reducing the capillary forces induced by solvents during evacuation, and specifically that solvents with sufficiently low surface tension could be used to activate either fragile or robust COFs without causing significant pore collapse.

Herein, we report a simple, efficient, and general COF activation approach involving washing with an ultralow surface tension solvent followed by vacuum drying. Similar to prior successful approaches that avoided pore collapse by minimizing capillary forces during drying 22,23 , our approach produced highquality structures with excellent crystallinities and high surface areas. We analyzed the activation of six different COFs, including both fragile and robust COFs (Figure 1), using a series of solvents varying in surface tension, from $11.9-72.8 \mathrm{mN} \mathrm{m}^{-1}$. Through analysis of the resulting sample crystallinity and surface areas, we found that the surface areas and crystallinities of fragile COFs depended strongly on the 
solvent used for activation. All COFs studied could be effectively activated using the ultralow surface tension solvent perfluorohexane (PFH), and the resulting surface areas were similar to those produced using supercritical $\mathrm{CO}_{2}$. PFH therefore provides a simple and effective route that is attractive for the scaleup and production of highly crystalline COFs.

\section{RESULTS AND DISCUSSION}

We chose six different imine-linked COF structures for analysis. Each COF was prepared through the imine condensation reaction between a multi-functional aldehyde and aromatic amine, shown in red and blue in Figure 1a, respectively. For example, TAPB-PDA COF was prepared by the imine condensation between TAPB and PDA ${ }^{22}$. The other five COFs chosen for analysis were TAPB-OH-PDA ${ }^{23,33,34}$ TAPB4F-PDA, TAPB-OMe-PDA ${ }^{23}$, TAPT-OMe-PDA ${ }^{35}$, and TAPB-BTCA ${ }^{36}$. Detailed synthesis procedures are provided in the Methods section and the Supporting Information.
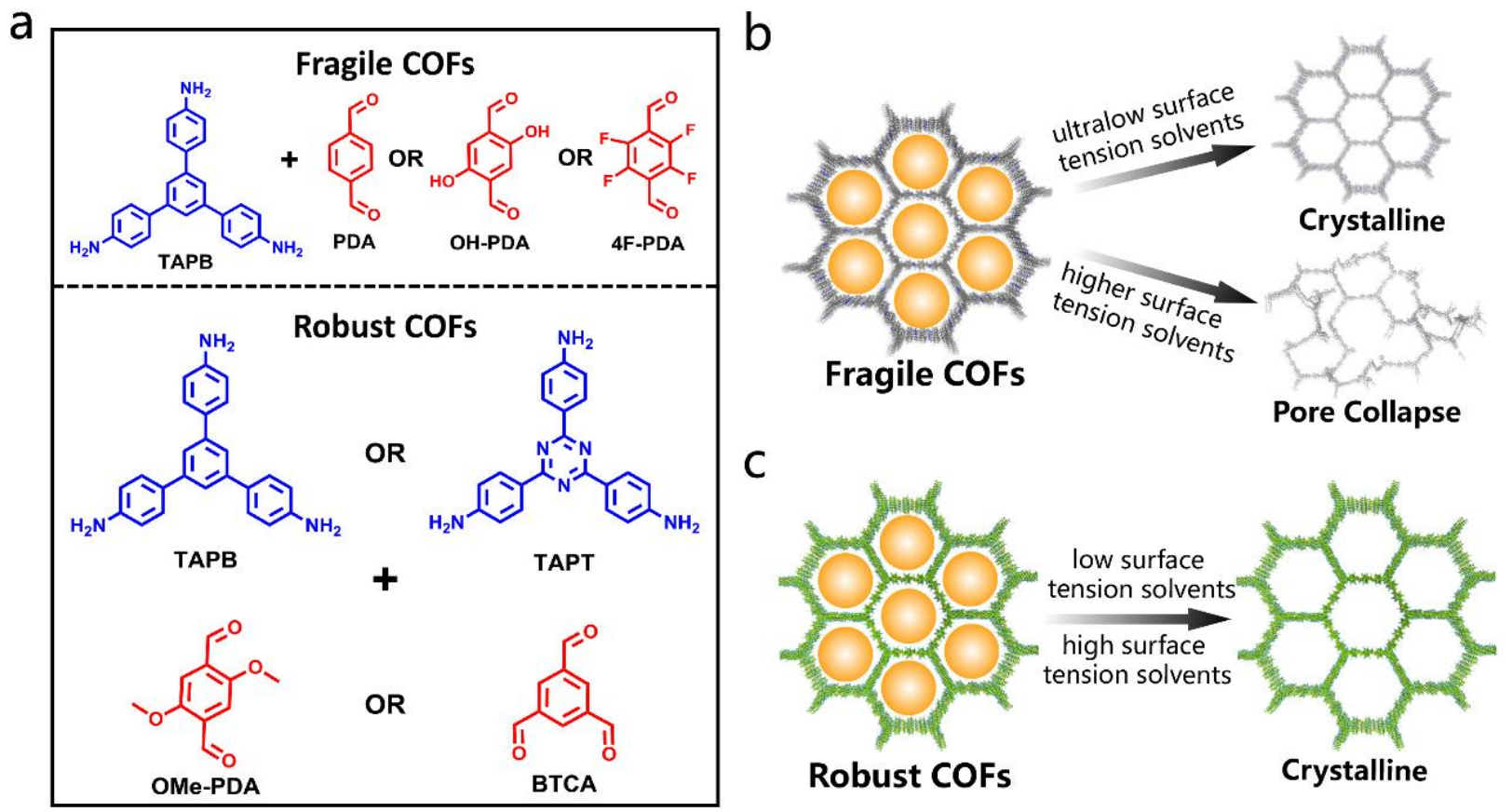

Figure 1. (a) Building blocks for different COFs studied in this work and (b, c) Schematic showing that successful activation depends on both COF chemistry and activation procedure.

These six COF structures were chosen for comparison with prior reports on activation of these materials and for understanding the effects of substituents and node symmetry. TAPB-PDA COF has been 
extensively studied and characterized ${ }^{23}$ and is an example of a fragile COF in which only gentle activation procedures can produce a highly crystalline sample ${ }^{22,23}$. TAPB-4F-PDA, TAPB-OH-PDA, TAPB-OMePDA and TAPT-OMe-PDA COFs provide insight into the effects of substituents on the dialdehyde linkers. Sick et al. ${ }^{22}$ and Feriante et al. ${ }^{23}$ found that COFs with substituents were more robust during activation due to stronger $\pi-\pi$ interactions between adjacent layers. Jiang et al. ${ }^{31,32}$ also reported that both fluorine atoms and hydroxy groups could increase the interlayer interactions either through selfcomplementary $\pi$-electronic forces ${ }^{32}$ or intralayer hydrogen bonds ${ }^{31}$. Additionally, these substituents can also increase steric hindrance and prevent structural distortion upon solvent removal. Very recently, Fang and coworkers ${ }^{37}$ reported the preparation of robust $3 \mathrm{D}$ COFs by engineering the steric hindrance in the COF structure. They showed that steric hindrance between substituents prevented pore shrinkage after removal of guest molecules. Steric hindrance has also be used to produce MOFs with higher stability towards external stimuli ${ }^{25}$. In this regard, we expected that larger substituents would produce greater steric hindrance in the COF structure, locking the structures more firmly and preventing pore collapse. TAPBBTCA COF combines C3 symmetric linkers and nodes, and we speculated that this combination would be more stable than the combination of $\mathrm{C} 3$ symmetric linkers and $\mathrm{C} 2$ symmetric nodes for the five other COFs studied. TAPB-BTCA COF also has a much smaller pore $\operatorname{size}^{36}(1.13 \mathrm{~nm})$ compared with TAPBPDA COF (pore size $3.3 \mathrm{~nm}^{22}$ ), which we expected would also contribute to improved stability.

We fist studied activation of the TAPB-PDA COF using a series of solvents varying in surface tension. TAPB-PDA COF was synthesized using a solvothermal method ${ }^{22}$ (see detailed procedure in the Supporting Information), washed with tetrahydrofuran (THF), and then immersed in a large excess of a selected solvent. The sample was then dried under vacuum overnight at $80^{\circ} \mathrm{C}$. The large excess of solvent used in the final wash step removed and replaced any residual solvents or guest molecules present during the solvothermal synthesis and determined the resulting capillary force during drying. We tested solvents ranging from low to high surface tensions $\left(11.9-72.8 \mathrm{mN} \mathrm{m}^{-1}\right.$, measured at $20^{\circ} \mathrm{C}^{38}$, see Table $\mathrm{S} 1$ in the Supporting Information). In order of low to high solvent surface tension, these were perfluorohexane (PHF), hexane, methanol, acetone, THF, dioxane, and dimethylformamide (DMF). 

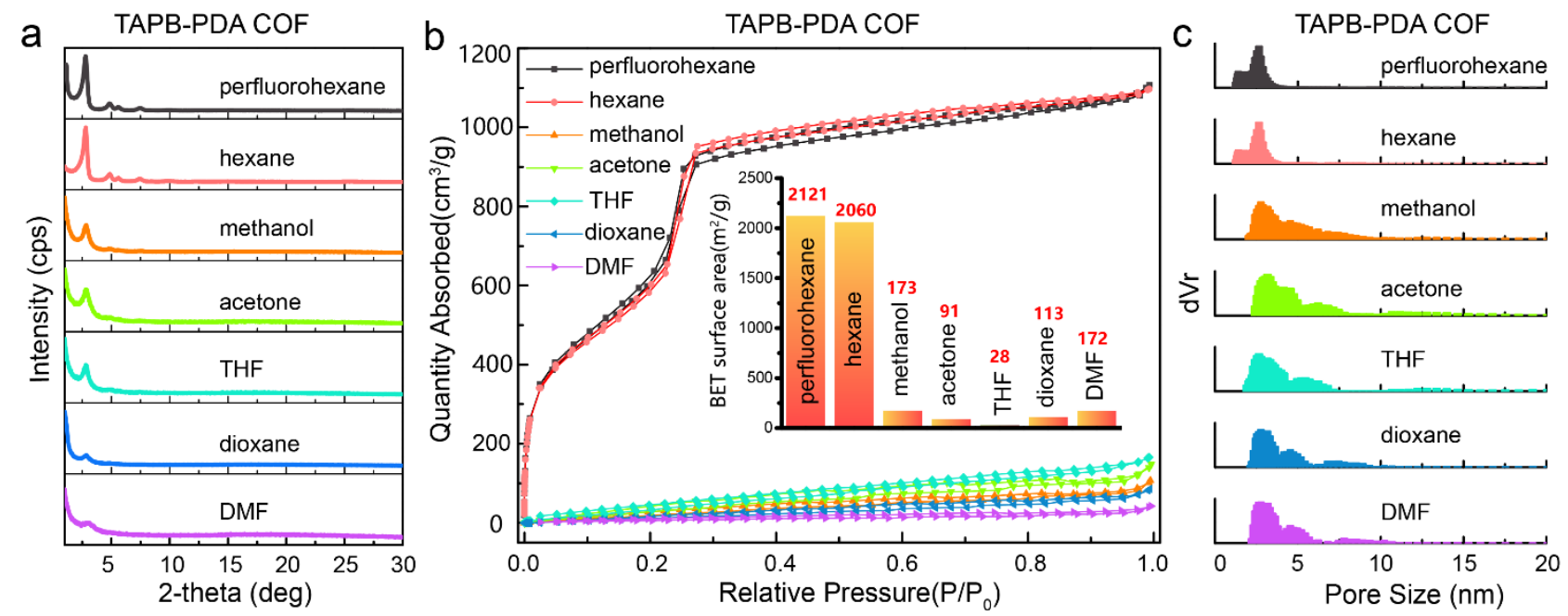

Figure 2. TAPB-PDA COF retained porous structure and crystallinity only after activation with low surface tension solvents as demonstrated by (a) PXRD spectra (b) nitrogen adsorption and desorption isotherms and (c) pore size distributions calculated using NLDFT methods. Insets in (b) show BET areas of TAPB-PDA COFs after activation with different solvents.

Each of these solvents was used to activate TAPB-PDA COF through solvent immersion followed by vacuum drying at $80^{\circ} \mathrm{C}$, and we additionally activated a sample of TAPB-PDA COF using supercritical $\mathrm{CO}_{2}\left(\mathrm{ScCO}_{2}\right)$. This was performed in a commercial critical point dryer (see Methods part).

As reflected in both powder X-ray diffraction (PXRD) and $\mathrm{N}_{2}$ sorption isotherms, the solvent chosen for washing the TAPB-PDA COF prior to drying had a significant impact on the measured crystallinities and Brunauer-Emmett-Teller (BET) surface areas (Figure 2). Samples washed with PFH and hexane retained excellent crystallinities and high surface areas comparable to samples produced using $\mathrm{ScCO}_{2}$ washing (Figure S1, Supporting Information). The sample washed with PFH had the highest measured surface area $\left(2121 \mathrm{~m}^{2} \mathrm{~g}^{-1}\right)$, slightly higher than that prepared using hexane $\left(2060 \mathrm{~m}^{2} \mathrm{~g}^{-1}\right)$ and comparable to the $\mathrm{ScCO}_{2}$ dried sample $\left(2097 \mathrm{~m}^{2} \mathrm{~g}^{-1}\right)$. We attributed the successful activation to the low surface tensions of PFH and hexanes, which minimized the capillary forces when removed under vacuum. When activation was performed using solvents of higher surface tension (greater than $18.7 \mathrm{mN} \mathrm{m}^{-1}$ ), the crystallinity and surface areas decreased substantially. The highest surface areas measured for these samples was $173 \mathrm{~m}^{2} \mathrm{~g}^{-1}$, reflecting significant pore collapse and shrinkage $\mathrm{e}^{22,23}$. These samples also lost crystallinity, as reflected in a strong reduction of the intensity of the $<100>$ peak, and PXRD spectra 
reflected short-range ordered structures for samples activated using methanol, acetone, THF, dioxane or DMF (Figure 2a). This attenuation in crystallinity and surface area reflects structural collapse of the pores caused by strong capillary forces when these higher surface tension solvents are removed under vacuum from the narrow COF pores ${ }^{24}$. These results are consistent with findings from previous reports $22,28,29,39,40$ which found substantially decreased BET surface areas after solvent exchange using dioxane ${ }^{22}$, toluene ${ }^{29}$ and $\mathrm{THF}^{28,39,40}$. Our results also show that the measured surface areas from solvents with very high surface tensions (dioxane and DMF) are similar to those with moderate surface tensions like methanol. This suggests that there is a threshold in solvent surface tension above which the porous and crystalline COF structure is completely disrupted.

For further insight into the role of solvent on the COF structure after activation, we estimated pore size distributions from the nitrogen sorption measurements using non-local density functional theory (NLDFT) (Figure 2c). TAPB-PDA COF activated with PFH or hexane retained a micro-porous structure, while activation with higher surface tension solvents damaged the micro-porous structure. This was also revealed directly from the nitrogen sorption isotherms (Figure $2 \mathrm{~b}$ ). Samples activated using PFH and hexane exhibited a sharp nitrogen adsorption increase at low pressures (relative pressure below 0.01) reflecting a microporous structure, while samples activated by more polar solvents did not show these features.

Next, we studied the impact of solvent exchange and activation across five other COFs to understand relationships between the molecular structures of the COFs and sensitivity to vacuum activations. Similar to the TAPB-PDA COFs, TAPB-4F-PDA lost crystallinity and surface area when activated with high surface tension solvents (Figures $3 \mathrm{a}$ and $4 \mathrm{a}$ ). Under activation using methanol, acetone, THF, dioxane and DMF, PXRD peaks declined sharply. Nitrogen adsorption and desorption tests after activation with THF or DMF revealed non-porous features and very low surface areas in these samples (Figure 4a). However, when PHF was used for activation, the sample retained a high degree of crystallinity (Figure 3a), and a high BET surface area of $819 \mathrm{~m}^{2} \mathrm{~g}^{-1}$, similar to that of $\mathrm{ScCO}_{2}$ activated samples $\left(919 \mathrm{~m}^{2} \mathrm{~g}^{-1}\right.$, see 
Supporting Information Figure S2). These results show that TAPB-4F PDA COFs exhibited similar fragility to TAPB-PDA COFs. While the F substituents have been shown to improve interlayer stability ${ }^{32}$, our results indicate these effects are not significant enough to stabilize the material during activation with polar solvents.
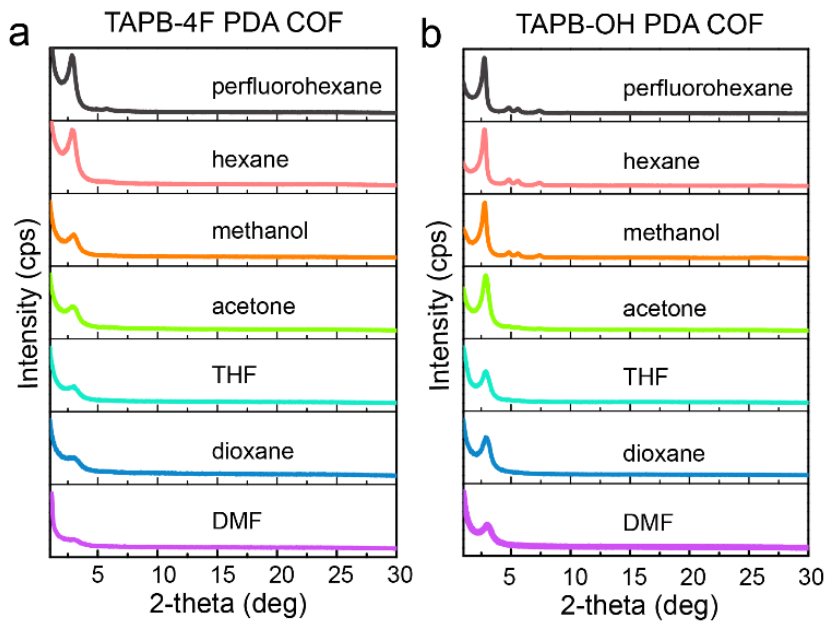

Figure 3. PXRD spectra of (a) TAPB-4F PDA COFs and (b) TAPB-OH PDA COFs activated using perfluorohexane, hexane, ethonal, acetone, THF, dioxane and DMF.

By comparison to TAPB-4F PDA, TAPB-OH-PDA COF was more stable to activation by different solvents. When activated using PFH or hexanes, the crystallinity was greater than when activated using other solvents (Figure 3b), and the BET surface area for the sample activated using PFH $\left(1649 \mathrm{~cm}^{2} \mathrm{~g}^{-1}\right.$, Figure $4 \mathrm{~b})$ was as large as that activated by $\mathrm{ScCO}_{2}\left(1644 \mathrm{~m}^{2} \mathrm{~g}^{-1}\right.$, Figure $\mathrm{S} 3$ in Supporting Information). The crystallinities and surface areas were only moderately reduced when using higher surface tension solvents. The crystallinity and long-range ordering was maintained when activated by methanol (Figure 3b), and TAPB-OH-PDA COF retained relatively high surface areas even under activation with very polar solvents THF $\left(712 \mathrm{~m}^{2} \mathrm{~g}^{-1}\right)$ and DMF $\left(517 \mathrm{~m}^{2} \mathrm{~g}^{-1}\right)$, as shown in Figure $4 \mathrm{~b}$ and the Supporting Information Figure S3. The porosity and crystallinity were lost, however, when activated with more polar solvents such as DMF.

TAPB-OH PDA exhibited semi-fragile behavior. The COF was more stable relative to TAPB-4F PDA and TAPB-PDA COF but still lost crystallinity and porosity when activated using polar solvents. 
The higher stability can be attributed to either stronger intralayer hydrogen bonding ${ }^{31}$ or stronger steric hinderance from larger $\mathrm{OH}$ substituents ${ }^{37}$. Activation using $\mathrm{PFH}$ as the activation produced samples with excellent crystallinities (Figure 3b) and a surface area as large as $1649 \mathrm{~m}^{2} \mathrm{~g}^{-1}$ (Figure 4b, Figure S3).

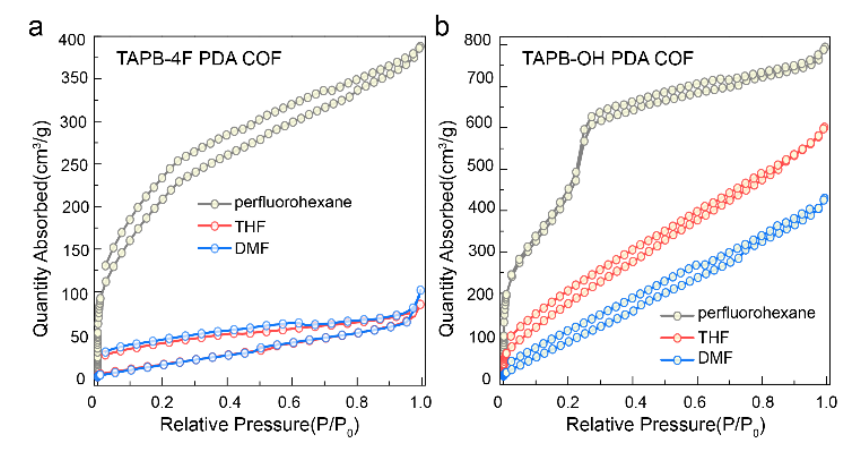

Figure 4. Nitrogen adsorption and desorption isotherms of (a) TAPB-4F PDA COFs and (b) TAPBOH PDA COFs activated using perfluorohexane, hexane, ethonal, acetone, THF, dioxane and DMF.

TAPB-OMe-PDA COF, TAPT-OMe PDA COF, and TAPB-BTCA COF were extremely robust and showed little sensitivity to activation by all solvents tested (Figure 5, Figures S 4, 5, 6, 26, and 33 in the Supporting Information). PXRD (Figure 5a) revealed excellent crystallinities and high surface areas (2200 2300 $\mathrm{m}^{2} \mathrm{~g}^{-1}$, Figure 5b, Figure S4) independent of solvent used for activation. The stability of TAPB-OMe-PDA and TAPT-OMe PDA-COFs can be attributed to strong interlayer interactions ${ }^{22,23}$ or steric hindrance ${ }^{37}$ caused by the large methoxy substitutent. The stability of TAPB-BTCA COF can potentially be attributed to more stable framework geometry and smaller pore size. Previous studies have shown that COFs with large pores can only be obtained through either activation by $\mathrm{ScCO}_{2}{ }^{20}$ or engineering steric hinderance in the linkers ${ }^{37}$ to avoid pore collapse.

For new COF chemistries, there is no reliable method to predict whether they will be robust, fragile, or semi-fragile. During workup of a COF sample, activation using polar solvents may lead to the incorrect conclusion that COF synthesis failed or that the material has poor crystallinity and porosity. This can be avoided by using a simple and gentle activation method. Washing with PFH followed by vacuum drying is a straightforward method to activation that does not require any specialized equipment or tools. Our 
results indicate that PFH provides a general and simple activation strategy for COFs, including fragile, semi-fragile and robust samples.
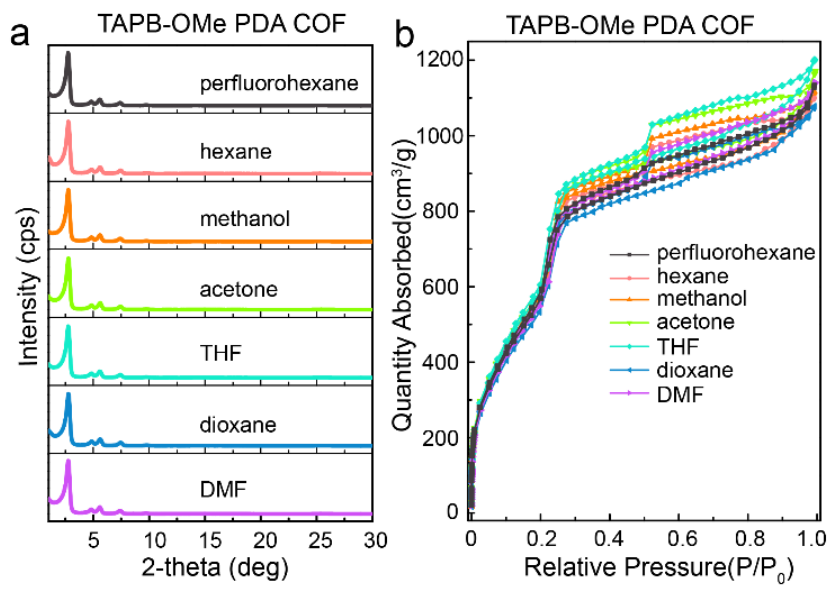

Figure 5. (a) PXRD spectra and (b) nitrogen adsorption and desorption isotherms of TAPB-OMePDA COFs activated using perfluorohexane, hexane, ethonal, acetone, THF, dioxane and DMF.

\section{CONCLUSIONS}

In summary, we demonstrated that ultralow surface tension solvent—perfluorohexane (PFH) can be used as an effective and efficient activation solvent for COFs. PXRD and nitrogen sorption test showed that activation using PFH produced COFs with excellent crystallinities and high surface areas comparable to $\mathrm{ScCO}_{2}$ activation. Due to the complexity and higher cost of $\mathrm{ScCO}_{2}$ activation ${ }^{22}$ or nitrogen flow activation $^{23}$, we anticipate our simple and efficient method can be widely used for COF activation under vacuum considering the recyclability of $\mathrm{PFH}$.

\section{MATERIALS AND METHODS}

Materials. All chemicals were purchased from commercial sources and used without further purification. 1,3,5-tris(4-aminophenyl)benzene (TAPB) were purchased from TCI America. Terephthaldehyde (PDA), 2,5-dimethoxyterephthalaldehyde (OMe-PDA), anhydrous dioxane and mesitylene were purchased from Sigma Aldrich. 2,3,5,6-tetrafluoroterephthalaldehyde (4F-PDA) and 
2,4,6-tris(4-aminophenyl)-1,3,5-triazine (TAPT) were purchased from AmBeed. 2,5dihydroxyterephthalaldehyde (OH-PDA) and benzene-1,3,5-tricarbaldehyde (BTCA) were purchased from Jilin Chinese Academy of Sciences - Yanshen Technology Co., Ltd. All other solvents used in this work were purchased from Fisher Scientific.

COF Synthesis. TAPB-PDA COF: TAPB (20.85 mg, $0.059 \mathrm{mmol})$ and PDA (12 mg, $0.089 \mathrm{mmol})$ were weighed and dissolved in a mixture of $1.6 \mathrm{~mL}$ dioxane and $0.4 \mathrm{~mL}$ mesitylene in a Pyrex tube directly. Before the tube being sealed, $0.2 \mathrm{~mL} 6 \mathrm{M}$ acetic acid was added and the solution was sonicated for 10 minutes. The sealed tubes were transferred into oven and heated to $120^{\circ} \mathrm{C}$ for 3 days. All of the products were separated and washed thoroughly using THF and ethanol. For vacuum drying, final solids will be washed and immersed in perfluorohexane, hexane, methanol, acetone, THF, dioxane, and DMF respectively to replace all other solvents. After filtration, samples were dried in vacuum oven under 80 ${ }^{\circ} \mathrm{C}$ overnight. For $\mathrm{ScCO}_{2}$ drying, samples were dried when they are wet in ethanol using the Leica EM CPD300 Critical Point Dryer. All different portions were kept the same reaction conditions except the activation procedures. Five other COFs were synthesized using the similar methods (Details are given in Supporting Information).

$\mathrm{ScCO}_{2}$ drying. Supercritical $\mathrm{CO}_{2}$ drying was conducted on a Leica EM CPD300 automated Critical Point Dryer. COF powders were thoroughly washed using THF and a large access of ethanol. Wetted COF powders were loaded in tea bags and then transferred into the dryer chambers with addition of appropriate amount of pure ethanol.

Characterizations. PXRD data were recorded on a Rigaku SmartLab XRD from $2 \theta=1^{\circ}$ up to $30^{\circ}$ with $0.02^{\circ}$ increment. Powder samples were leveled flat on zero background sample holders. Fouriertransform infrared spectroscopy (FTIR) of all solid samples were tested using a ThermoNicolet iS10 FTIR spectrometer with a diamond ATR attachment and are uncorrected. Nitrogen sorption measurements were conducted on Quantachrome Autosorb-iQ-MP/Kr BET Surface Analyzer. All samples were dried 
and degassed at $80{ }^{\circ} \mathrm{C}$ for $12 \mathrm{~h}$ and backfilled with $\mathrm{N}_{2}$. BET surface areas were determined using BET adsorption models included in the instrument software (ASiQwin version 5.2).

\section{ASSOCIATED CONTENT}

\section{Supporting Information}

Solvent surface tensions, additional BET surface area measurements, detailed synthesis methods, and additional COF characterization data.

\section{AUTHOR INFORMATION}

Corresponding Author

*(R.V.) E-mail: rafaelv@rice.edu.

\section{ORCID}

Rafael Verduzco: 0000-0002-3649-3455

\section{Notes}

The authors declare no competing financial interest.

\section{ACKNOWLEDGMENT}

The authors acknowledge financial support from the Army Research Laboratory (W911NF-18-2-0062) and the Welch Foundation for Chemical Research (C-1888). The authors also acknowledge Shared Equipment Authority at Rice University for access and utilization of characterization instruments.

\section{REFERENCES}

(1) Kandambeth, S.; Dey, K.; Banerjee, R. Covalent Organic Frameworks: Chemistry beyond the Structure. J. Am. Chem. Soc. 2019, 141 (5), 1807-1822. 
(2) Ding, S.-Y.; Wang, W. Covalent Organic Frameworks (COFs): From Design to Applications. Chem. Soc. Rev. 2012, 42 (2), 548-568.

(3) Zhou, H.-C.; Long, J. R.; Yaghi, O. M. Introduction to Metal-Organic Frameworks. Chem. Rev. 2012, $112(2), 673-674$.

(4) Weckhuysen, B. M.; Yu, J. Recent Advances in Zeolite Chemistry and Catalysis. Chem. Soc. Rev. 2015, 44 (20), 7022-7024.

(5) Furukawa, H.; Yaghi, O. M. Storage of Hydrogen, Methane, and Carbon Dioxide in Highly Porous Covalent Organic Frameworks for Clean Energy Applications. J. Am. Chem. Soc. 2009, 131 (25), $8875-8883$.

(6) Li, Z.; Feng, X.; Zou, Y.; Zhang, Y.; Xia, H.; Liu, X.; Mu, Y. A 2D Azine-Linked Covalent Organic Framework for Gas Storage Applications. Chem. Commun. 2014, 50 (89), 13825-13828.

(7) Yuan, S.; Li, X.; Zhu, J.; Zhang, G.; Puyvelde, P. V.; Bruggen, B. V. der. Covalent Organic Frameworks for Membrane Separation. Chem. Soc. Rev. 2019.

(8) Fan, H.; Mundstock, A.; Feldhoff, A.; Knebel, A.; Gu, J.; Meng, H.; Caro, J. Covalent Organic Framework-Covalent Organic Framework Bilayer Membranes for Highly Selective Gas Separation. J. Am. Chem. Soc. 2018, 140 (32), 10094-10098.

(9) Gonçalves, R. S. B.; de Oliveira, A. B. V.; Sindra, H. C.; Archanjo, B. S.; Mendoza, M. E.; Carneiro, L. S. A.; Buarque, C. D.; Esteves, P. M. Heterogeneous Catalysis by Covalent Organic Frameworks (COF): $\mathrm{Pd}(\mathrm{OAc}) 2 @ \mathrm{COF}-300$ in Cross-Coupling Reactions. ChemCatChem 2016, 8 (4), 743-750.

(10) Sharma, R. K.; Yadav, P.; Yadav, M.; Gupta, R.; Rana, P.; Srivastava, A.; Zbořil, R.; Varma, R. S.; Antonietti, M.; Gawande, M. B. Recent Development of Covalent Organic Frameworks (COFs): Synthesis and Catalytic (Organic-Electro-Photo) Applications. Mater. Horiz. 2020, 7 (2), 411-454.

(11) Ding, S.-Y.; Gao, J.; Wang, Q.; Zhang, Y.; Song, W.-G.; Su, C.-Y.; Wang, W. Construction of Covalent Organic Framework for Catalysis: Pd/COF-LZU1 in Suzuki-Miyaura Coupling Reaction. J. Am. Chem. Soc. 2011, 133 (49), 19816-19822. 
(12) Ding, S.-Y.; Dong, M.; Wang, Y.-W.; Chen, Y.-T.; Wang, H.-Z.; Su, C.-Y.; Wang, W. ThioetherBased Fluorescent Covalent Organic Framework for Selective Detection and Facile Removal of Mercury(II). J. Am. Chem. Soc. 2016, 138 (9), 3031-3037.

(13) Sun, Q.; Aguila, B.; Perman, J.; Earl, L. D.; Abney, C. W.; Cheng, Y.; Wei, H.; Nguyen, N.; Wojtas, L.; Ma, S. Postsynthetically Modified Covalent Organic Frameworks for Efficient and Effective Mercury Removal. J. Am. Chem. Soc. 2017, 139 (7), 2786-2793.

(14) Fan, H.; Gu, J.; Meng, H.; Knebel, A.; Caro, J. High-Flux Membranes Based on the Covalent Organic Framework COF-LZU1 for Selective Dye Separation by Nanofiltration. Angewandte Chemie International Edition 2018, 57 (15), 4083-4087.

(15) Corcos, A. R.; Levato, G. A.; Jiang, Z.; Evans, A. M.; Livingston, A. G.; Mariñas, B. J.; Dichtel, W. R. Reducing the Pore Size of Covalent Organic Frameworks in Thin-Film Composite Membranes Enhances Solute Rejection. ACS Materials Lett. 2019, 1 (4), 440-446.

(16) Segura, J. L.; Mancheño, M. J.; Zamora, F. Covalent Organic Frameworks Based on Schiff-Base Chemistry: Synthesis, Properties and Potential Applications. Chem. Soc. Rev. 2016, 45 (20), 5635 5671.

(17) Campbell, N. L.; Clowes, R.; Ritchie, L. K.; Cooper, A. I. Rapid Microwave Synthesis and Purification of Porous Covalent Organic Frameworks. Chem. Mater. 2009, 21 (2), 204-206.

(18) Wei, H.; Chai, S.; Hu, N.; Yang, Z.; Wei, L.; Wang, L. The Microwave-Assisted Solvothermal Synthesis of a Crystalline Two-Dimensional Covalent Organic Framework with High $\mathrm{CO}_{2}$ Capacity. Chem. Commun. 2015, 51 (61), 12178-12181.

(19) Yang, S.-T.; Kim, J.; Cho, H.-Y.; Kim, S.; Ahn, W.-S. Facile Synthesis of Covalent Organic Frameworks COF-1 and COF-5 by Sonochemical Method. RSC Advances 2012, 2 (27), 1017910181.

(20) Matsumoto, M.; Dasari, R. R.; Ji, W.; Feriante, C. H.; Parker, T. C.; Marder, S. R.; Dichtel, W. R. Rapid, Low Temperature Formation of Imine-Linked Covalent Organic Frameworks Catalyzed by Metal Triflates. J. Am. Chem. Soc. 2017, 139 (14), 4999-5002. 
(21) Karak, S.; Kandambeth, S.; Biswal, B. P.; Sasmal, H. S.; Kumar, S.; Pachfule, P.; Banerjee, R. Constructing Ultraporous Covalent Organic Frameworks in Seconds via an Organic Terracotta Process. J. Am. Chem. Soc. 2017, 139 (5), 1856-1862.

(22) Sick, T.; Rotter, J. M.; Reuter, S.; Kandambeth, S.; Bach, N. N.; Döblinger, M.; Merz, J.; Clark, T.; Marder, T. B.; Bein, T.; Medina, D. D. Switching on and off Interlayer Correlations and Porosity in 2D Covalent Organic Frameworks. J. Am. Chem. Soc. 2019, 141 (32), 12570-12581.

(23) Feriante, C. H.; Jhulki, S.; Evans, A. M.; Dasari, R. R.; Slicker, K.; Dichtel, W. R.; Marder, S. R. Rapid Synthesis of High Surface Area Imine-Linked 2D Covalent Organic Frameworks by Avoiding Pore Collapse During Isolation. Advanced Materials 2020, 32 (2), 1905776.

(24) Mondloch, J. E.; Karagiaridi, O.; Farha, O. K.; Hupp, J. T. Activation of Metal-Organic Framework Materials. CrystEngComm 2013, 15 (45), 9258-9264.

(25) Nelson, A. P.; Farha, O. K.; Mulfort, K. L.; Hupp, J. T. Supercritical Processing as a Route to High Internal Surface Areas and Permanent Microporosity in Metal-Organic Framework Materials. $J$. Am. Chem. Soc. 2009, 131 (2), 458-460.

(26) Ma, J.; Kalenak, A. P.; Wong-Foy, A. G.; Matzger, A. J. Rapid Guest Exchange and Ultra-Low Surface Tension Solvents Optimize Metal-Organic Framework Activation. Angewandte Chemie International Edition 2017, 56 (46), 14618-14621.

(27) Ma, L.; Jin, A.; Xie, Z.; Lin, W. Freeze Drying Significantly Increases Permanent Porosity and Hydrogen Uptake in 4,4-Connected Metal-Organic Frameworks. Angewandte Chemie International Edition 2009, 48 (52), 9905-9908.

(28) Xu, Q.; Tao, S.; Jiang, Q.; Jiang, D. Designing Covalent Organic Frameworks with a Tailored Ionic Interface for Ion Transport across One-Dimensional Channels. Angewandte Chemie International Edition 2020, 59 (11), 4557-4563.

(29) Smith, B. J.; Overholts, A. C.; Hwang, N.; Dichtel, W. R. Insight into the Crystallization of Amorphous Imine-Linked Polymer Networks to 2D Covalent Organic Frameworks. Chem. Commun. 2016, 52 (18), 3690-3693. 
(30) Ascherl, L.; Sick, T.; Margraf, J. T.; Lapidus, S. H.; Calik, M.; Hettstedt, C.; Karaghiosoff, K.; Döblinger, M.; Clark, T.; Chapman, K. W.; et al. Molecular Docking Sites Designed for the Generation of Highly Crystalline Covalent Organic Frameworks. Nature Chemistry 2016, 8 (4), $310-316$.

(31) Chen, X.; Addicoat, M.; Jin, E.; Zhai, L.; Xu, H.; Huang, N.; Guo, Z.; Liu, L.; Irle, S.; Jiang, D. Locking Covalent Organic Frameworks with Hydrogen Bonds: General and Remarkable Effects on Crystalline Structure, Physical Properties, and Photochemical Activity. J. Am. Chem. Soc. 2015, $137(9), 3241-3247$.

(32) Chen, X.; Addicoat, M.; Irle, S.; Nagai, A.; Jiang, D. Control of Crystallinity and Porosity of Covalent Organic Frameworks by Managing Interlayer Interactions Based on Self-Complementary

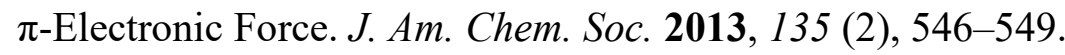

Li, Z.; Liu, Z.-W.; Li, Z.; Wang, T.-X.; Zhao, F.; Ding, X.; Feng, W.; Han, B.-H. Defective 2D Covalent Organic Frameworks for Postfunctionalization. Advanced Functional Materials 2020, 30 (10), 1909267.

(34) Biswal, B. P.; Kandambeth, S.; Chandra, S.; Shinde, D. B.; Bera, S.; Karak, S.; Garai, B.; Kharul, U. K.; Banerjee, R. Pore Surface Engineering in Porous, Chemically Stable Covalent Organic Frameworks for Water Adsorption. J. Mater. Chem. A 2015, 3 (47), 23664-23669.

(35) Mullangi, D.; Chakraborty, D.; Pradeep, A.; Koshti, V.; Vinod, C. P.; Panja, S.; Nair, S.; Vaidhyanathan, R. Highly Stable COF-Supported $\mathrm{Co} / \mathrm{Co}(\mathrm{OH})_{2}$ Nanoparticles Heterogeneous Catalyst for Reduction of Nitrile/Nitro Compounds under Mild Conditions. Small 2018, 14 (37), 1801233.

Li, X.; Gao, Q.; Aneesh, J.; Xu, H.-S.; Chen, Z.; Tang, W.; Liu, C.; Shi, X.; Adarsh, K. V.; Lu, Y.; Loh, K. P. Molecular Engineering of Bandgaps in Covalent Organic Frameworks. Chem. Mater. 2018, 30 (16), 5743-5749. 
(37) Wang, Y.; Liu, Y.; Li, H.; Guan, X.; Xue, M.; Yan, Y.; Valtchev, V.; Qiu, S.; Fang, Q. ThreeDimensional Mesoporous Covalent Organic Frameworks through Steric Hindrance Engineering. $J$. Am. Chem. Soc. 2020, 142 (8), 3736-3741.

(38) Surface tension values of some common test liquids for surface energy analysis http://www.surface-tension.de/

(39) Xu, Q.; Tao, S.; Jiang, Q.; Jiang, D. Ion Conduction in Polyelectrolyte Covalent Organic Frameworks. J. Am. Chem. Soc. 2018, 140 (24), 7429-7432.

(40) Xu, H.; Tao, S.; Jiang, D. Proton Conduction in Crystalline and Porous Covalent Organic Frameworks. Nature Materials 2016, 15 (7), 722-726. 